\title{
DETECTION OF BUILT-UP AREAS USING POLARIMETRIC SYNTHETIC APERTURE RADAR DATA AND HYPERSPECTRAL IMAGE
}

\author{
R. Bordbari ${ }^{\text {a, } *, \text { Y. Maghsoudi }}{ }^{\text {a }}$, M. Salehi ${ }^{\text {a }}$

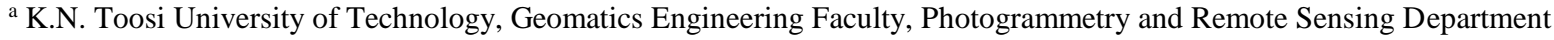

KEY WORDS: Polarimetric synthetic aperture radar (POLSAR), polarimetric target decomposition (PTD), hyperspectral image (HIS), target detection, correlation coefficient

\begin{abstract}
:
Polarimetric synthetic aperture radar (POLSAR) is an advantageous data for information extraction about objects and structures by using the wave scattering and polarization properties. Hyperspectral remote sensing exploits the fact that all materials reflect, absorb, and emit electromagnetic energy, at specific wavelengths, in distinctive patterns related to their molecular composition. As a result of their fine spectral resolution, Hyperspectral image (HIS) sensors provide a significant amount of information about the physical and chemical composition of the materials occupying the pixel surface. In target detection applications, the main objective is to search the pixels of an HSI data cube for the presence of a specific material (target). In this research, a hierarchical constrained energy minimization (hCEM) method using 5 different adjusting parameters has been used for target detection from hyperspectral data. Furthermore, to detect the built-up areas from POLSAR data, building objects discriminated from surrounding natural media presented on the scene using Freeman polarimetric target decomposition (PTD) and the correlation coefficient between co-pol and cross-pol channels. Also, target detection method has been implemented based on the different polarization basis for using the more information. Finally a majority voting method has been used for fusing the target maps. The polarimetric image C-band SAR data acquired by Radarsat-2, over San Francisco Bay area was used for the evaluation of the proposed method.
\end{abstract}

\section{INTRODUCTION}

In this paper, we consider how combination of polarimetric synthetic aperture radar (PolSAR) data and hyperspectral images can be used to enhance the detection of targets (built-up area). Hyperspectral imaging (HSI) sensors collect data that can be represented by a three-dimensional data cube. For each pixel within a hyperspectral image, a continuous spectrum is sampled and can be used to identify materials by their reflectance. One shortcoming of HSI is that it provides no surface penetration. To overcome these limitations and enhance HSI system performance, we fuse HSI data with PolSAR sensor data. In counter camouflage, concealment, and deception applications, HSI data can be used to identify ground cover and surface material, and a PolSAR data can determine if any threat objects are under concealment. Because PolSAR and HSI sensors exploit the different phenomenology, their detection capabilities complement each other.

PolSAR penetrates foliage and detects targets under tree canopy, but has significant clutter returns from trees. HSI, on the other hand, is capable of subpixel detection and material identification. Both SAR and HSI systems may suffer substantial false-alarm and missed detection rates because of their respective background clutter, but we expect that combining SAR and HSI data will greatly enhance detection and identification performance.

\section{POLARIMETRIC DETECTION}

\subsection{Polarimetric SAR Target Detection}

The strategic advantage of SAR in target detection is the possibility to monitor under foliage. In PolSAR images, the main feature of target is a relatively large backscattering signal, which is usually brighter in comparison with the clutter. Generally, some statistical tests on the intensity of the clutter or the polarimetric target decompositions (PTD) based on the physics concepts (Yamaguchi, et al. 2005) have been applied to separate the targets from the background.

Several detectors were proposed in the recent years. Some of them exploit the different polarimetric channels as independent measurements of the same scene (Rey 2002). Another class of polarimetric detectors adds some physical rationale exploiting knowledge regarding the scattering. The idea behind these methodologies is that the differences between clutter and targets can be magnified if some specific aspects of the polarimetric return are observed. In this second category, there are algorithms with a detection role based on some rationale linked to the physical behavior of the clutter (Nunziata, Migliaccio et al. 2012)

The built-up areas could be estimated by many methods ( $\mathrm{T}$. Moriyama et al. 2005, L. Zhang et al 2008, S. Guillaso et al. 2003, Guillaso et al. 2005). In this paper, PTD and polarimetric correlation coefficient are used to quickly estimate built-up area as shown in Fig. 1. The structure of building is like a dihedral corner reflector, so these areas have strong double-bounce scattering component. The double-bounce scattering component can be estimated by using the PTD.

\footnotetext{
* Corresponding author
} 
The measured coherency matrix can be represented as the sum of several scattering components using PTD (Moriyama et al. 2005). For example, the coherency matrix is decomposed into the three scattering mechanisms corresponding to odd-bounce, even-bounce, and volume scattering for PolSAR data in built-up area.

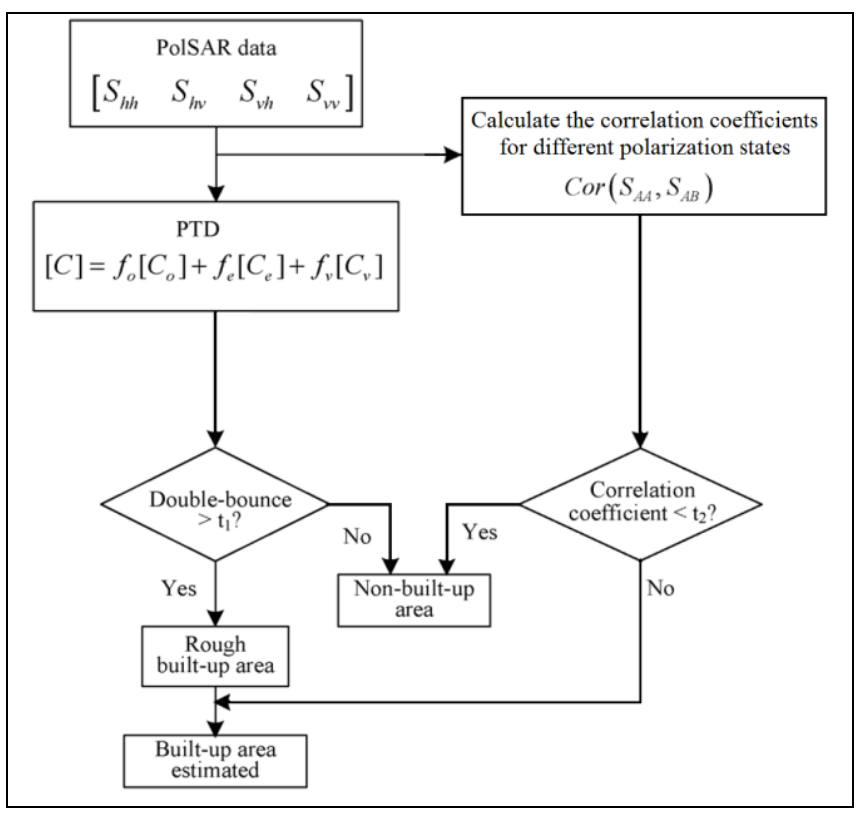

Fig. 1. The flow chart of detection of built-up area.

The volume scattering represents the remaining component besides odd-bounce and even-bounce scattering in built-up area.

$$
T=f_{0}\left[T_{0}\right]+f_{e}\left[T_{e}\right]+f_{v}\left[T_{v}\right]
$$

where $f_{0}, f_{e}$ and $f_{v}$ represent the weight of odd-bounce scattering, even-bounce scattering, and volume scattering, respectively. $\left[T_{0}\right],\left[T_{e}\right]$ and $\left[T_{v}\right]$ represent the corresponding coherency base respectively.

$$
\begin{aligned}
& {\left[T_{0}\right]=\frac{1}{2}\left[\begin{array}{ccc}
|\beta+1|^{2} & (\beta+1)(\beta-1)^{*} & 0 \\
(\beta-1)(\beta+1)^{*} & |\beta-1|^{2} & 0 \\
0 & 0 & 0
\end{array}\right]} \\
& {\left[T_{e}\right]=\frac{1}{2}\left[\begin{array}{ccc}
|\alpha+1|^{2} & (\alpha+1)(\alpha-1)^{*} & 0 \\
(\alpha-1)(\alpha+1)^{*} & |\alpha-1|^{2} & 0 \\
0 & 0 & 0
\end{array}\right]} \\
& {\left[T_{v}\right]=\frac{1}{2}\left[\begin{array}{ccc}
|\gamma+1|^{2} & (\gamma+1)(\gamma-1)^{*} & 2(\gamma+1) \rho^{*} \\
(\gamma-1)(\gamma+1)^{*} & |\gamma-1|^{2} & 2(\gamma-1) \rho^{*} \\
2 \rho(\gamma+1)^{*} & 2 \rho(\gamma-1)^{*} & 4|\rho|^{2}
\end{array}\right]}
\end{aligned}
$$

where $\beta$ is a ratio of $\mathrm{HH}$ backscatter to VV backscatter in oddbounce scattering and $\alpha$ is a coefficient similar to $\beta$ in evenbounce scattering. $\gamma$ and $\rho$ are the ratios of $\mathrm{HH}$ and $\mathrm{HV}$ backscatters to VV backscatter in volume scattering respectively.

But a ground-trunk interaction for forests is also like a dihedral corner reflector in $\mathrm{C}$ and $\mathrm{L}$ bands. To detect the refined built-up areas, the different scattering characteristics between natural distributed areas and built-up areas is used. The main point of difference is polarimetric correlation coefficient, because the reflection symmetry condition, $\left\langle S_{h h} S_{h v}^{*}\right\rangle=\left\langle S_{v v} S_{h v}^{*}\right\rangle \approx 0$ does not hold for built-up areas. The correlation coefficient between co- and cross-polarized channels is defined by [6]

$\operatorname{Cor}\left(S_{p p}, S_{q q}\right)=\frac{\left|\left\langle S_{p p} S_{p q}^{*}\right\rangle\right|}{\sqrt{\left\langle S_{p p} S_{p p}^{*}\right\rangle\left\langle S_{p q} S_{p q}^{*}\right\rangle}}(p, q=h, v, p \neq q)$

If the correlation coefficient $\operatorname{Cor}\left(S_{A A}, S_{A B}\right)$ is close to one in a test area, this area can be seen as a built-up area. As shown in Fig. 1, we used different polarization states (different ellipticities and orientation angles) to calculate several correlation coefficients.

Polarization refers to the alignment and regularity of the electric field component of the Electromagnetic wave. The path of the end point of the Electric wave vector traces out an ellipse in its general form as shown in Fig.2. The size of the ellipse is proportional to the amplitude of the wave. The shape can be characterized by two geometrical polarization parameters, the ellipticity $\tau$ varying from -45 to +45 and the orientation angle $\varphi$ varying from 0 to 180 . The electric field of a monochromatic plane wave propagating in the $\mathrm{z}$-direction can be represented by a two component vector in any polarization basis. This can be expressed in terms of a complex polarization vector.

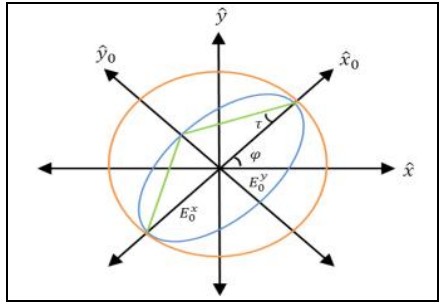

Fig. 2. Polarimetric ellipse

$$
\underline{E}=\left[\begin{array}{c}
E_{H} \\
E_{V}
\end{array}\right]=\left[\begin{array}{l}
\alpha_{H^{*}} \exp \left(j \delta_{H}\right) \\
\alpha_{V^{*}} \cdot \exp \left(j \delta_{V}\right)
\end{array}\right]
$$

Rough built-up area can be estimated firstly by setting a threshold value $t_{1}$ of double-bounce scattering component, then refined built-up area can be obtained by setting a threshold value $t_{2}$ of correlation coefficient to rough built-up area. In this paper, the values of $t_{1}$ and $t_{2}$ are the mean value of doublebounce scattering component and correlation coefficient.

\section{HYPERSPECTRAL DETECTION}

\subsection{Detection methods overview}

Recently, target detection has attracted considerable interest in many hyperspectral remote sensing applications, such as agriculture, forestry, geology, and defence. In fact, the aim of target detection is to identify targets, rare pixels with known spectral signatures. Over the last two decades, several detection algorithm have been developed using statistical, physical, or heuristic approaches (Manolakis and Shaw 2002, Manolakis, Marden et al. 2003, Nasrabadi 2014). Most algorithms are based on second-order statistics to construct detector, such as the matched filter (MF) (Manolakis, Marden et al. 2003), the constrained energy minimization (CEM) (Farrand and Harsanyi 1997) and the adaptive coherence estimator (ACE) (Kraut, Scharf et al. 2001, Manolakis, Marden et al. 2003).

In 2015, Zou and Shi instead of refining the target spectrum directly similar to (T. Wang et al. 2014, X. Fan et al. 2011), 
built a new hierarchical CEM (hCEM) to suppress the variational background spectra while preserving the targets. In here, we use the hCEM method with the purpose of improving the performance of traditional CEM detector. Since the classical CEM detector, in some special cases, cannot completely push out the targets and suppress the backgrounds in one round of filtering process, we filter the data for several times to solve this problem. In hCEM method, the CEM detectors of different layers are linked in series. After each layer's detection, some background spectra are suppressed by a nonlinear function based on the output of the detector. Then, the transformed spectra are forward sent to the next layer's detector, until the CEM detector's output converges to a constant. Suppressing the undesired backgrounds makes the CEM detector better concentrate on the hard-detected targets. In this way, the performance of the detector will be gradually enhanced layer by layer.

\subsection{Brief Introduction to CEM}

Consider a hyperspectral image with $\mathrm{N}$ spectral vectors and $\mathrm{L}$ bands: $x_{i} \in \mathbb{R}^{L \times 1}, i=1,2, \ldots, N$. All spectra of the hyperspectral image can be arranged in an $L \times N$ matrix as $X=\left[x_{1}, x_{2}, \ldots, x_{N}\right]$. The aim of CEM algorithm is to design an optimal finite impulse response (FIR) filter, specified by the vector $w=\left[w_{1}, w_{2}, \ldots, w_{L}\right]^{T}$. The average output energy from all the pixel vectors can be represented as

$\frac{1}{N}\|y\|_{2}^{2}=\frac{1}{N}\left\|w^{T} X\right\|_{2}^{2}=\frac{1}{N} w^{T} X X^{T} w=w^{T} R w$

where $R=(1 / N) X X^{T}$ represents the correlation matrix, and $y=\left[y_{1}, y_{2}, \ldots, y_{N}\right] \in \mathbb{R}^{1 \times N}$ represents the output of the detector. The CEM designs an FIR filter, which minimizes the total output energy, subject to a constraint that the filter's response to $d$ is a constant (e.g., $\left.w^{T} d=1\right)$ as follows:

$$
\begin{gathered}
\min \\
\text { s.t. } \\
w^{T} d=1
\end{gathered}
$$

where $d$ is a prechosen target spectrum and can be obtained by averaging different target spectral vectors of a certain material in one hyperspectral image. The solution of the aforementioned optimization problem is given in (W. H. Farrand et al, 1997), which is

$$
\begin{aligned}
& y=\left(w^{*}\right)^{T} X \\
= & \frac{d^{T} R^{-1}}{d R^{-1} d^{T}} X .
\end{aligned}
$$

Usually, the target pixels will get large value of outputs, while the background pixels will get small ones. Finally, each element of $y$ is compared with a fix threshold. If the output value is higher than the threshold, we decide the target present in the corresponding pixel; otherwise, we decide there is a target absent.

\section{3 hCEM}

In hCEM method, we perform a transformation on the spectra for beneficial target detection. The traditional CEM detector is a single-layer detector, while the proposed hCEM detector consists of different layers of traditional CEM detectors, and the detectors of different layers are linked in series. After each layer of detection, the background spectra are suppressed (reduce its magnitude while keeping its direction in the spectral space) based on the current layer's output score. The CEM detector is constructed based on the correlation matrix R, while the hCEM detector is constructed based on the corresponded revised correlation matrix. Since the revised correlation matrix contains more information of the hard-detected spectra, the hCEM could have better concentration on those hard-detected pixels (Zou and Shi, 2015).

Now, consider the kth layer. The CEM output of this layer can be represented as

$$
y^{k}=\frac{R_{k}^{-1} d}{d^{T} R_{k}^{-1} d} X^{k}
$$

where $X^{k}$ and $R^{k}$ represent the spectral matrix and the correlation matrix of the kth layer, respectively. Then, each spectral vector $x_{i}^{k}$ is transformed by multiplying a nonnegative number $q\left(y_{i}^{k}\right)$ based on its output score as follows:

$$
x_{i}^{k+1}=q\left(y_{i}^{k}\right) x_{i}^{k}
$$

where a nonlinear function $q(t): \mathbb{R}^{1} \rightarrow \mathbb{R}^{1}$ is used to impose on the spectral vector $x_{i}^{k}$. We consider this function as a "softthreshold" operation: hold the spectrum $x_{i}$ whose output score is large, while suppress the spectrum $x_{j}$ whose output score is small. In this way, the undesired background spectra are gradually suppressed after each layer's detection, while the target spectra will remain unchanged. In this paper, the nonlinear suppression function is defined as follows:

$$
q(t)=\left\{\begin{array}{cc}
q(t)=1-e^{-\lambda t,} & t \geq 0 \\
0, & t<0
\end{array}\right.
$$

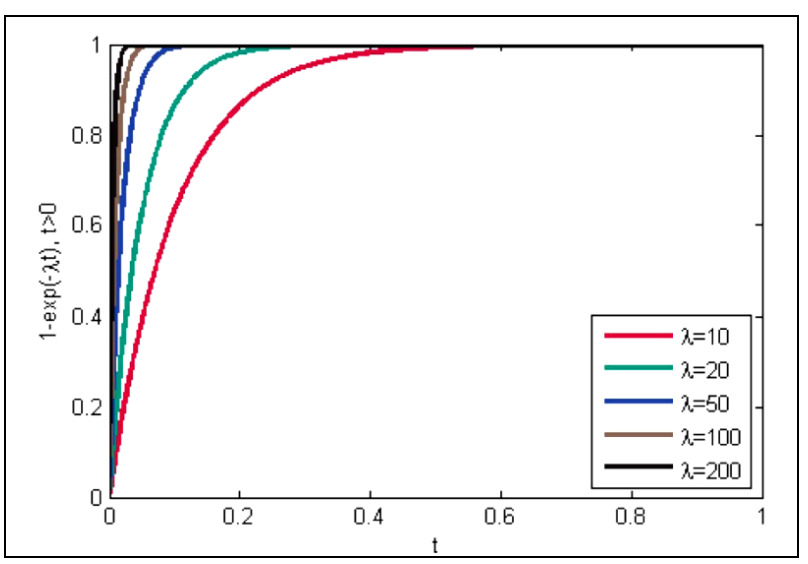

Fig. 3. Shape of the nonlinear suppression function $q(t)=1-e^{-\lambda t}, t \geq 0$ with different choices of $\lambda$.

where $\lambda$ is a positive parameter to adjust the shape of the function (10). Fig. 3 shows shape of function (10) under different choices of $\lambda$. Finally, the target spectra and the transformed background spectra will be used to construct the new CEM detector in the $(k+1)$ th layer. The aforementioned steps will be repeated until the output $y^{k}$ converges to a constant. In this paper, we calculate $\delta_{k}$ the error of the average output energy of the current layer and the previous layer, as follows:

$$
\delta_{k}=\frac{1}{N}\left\|y^{k}\right\|_{2}^{2}-\frac{1}{N}\left\|y^{k-1}\right\|_{2}^{2} .
$$


If $\delta_{k}<\varepsilon$ ( $\varepsilon$ refers to a small positive number), the iteration will be stopped.

\section{EXPERIMENTAL RESULTS AND DECISION FUSION}

\subsection{Study area}

The Radarsat-2 C-band full polarized image and Hyperion hyperspectral image of San Francisco, in northern California, USA, are used for the buildings detection. The nominal slant range resolution of POLSAR data is $11.1 \mathrm{~m}$ at near range to $10.5 \mathrm{~m}$ at far range. The spatial resolution of hyperspectral image is $30 \mathrm{~m} \times 30 \mathrm{~m}$.

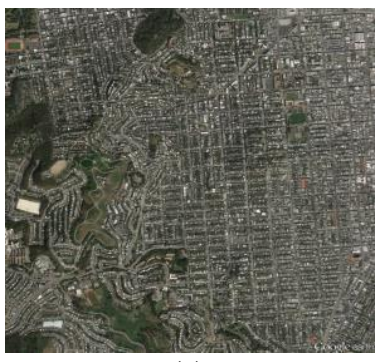

(a)

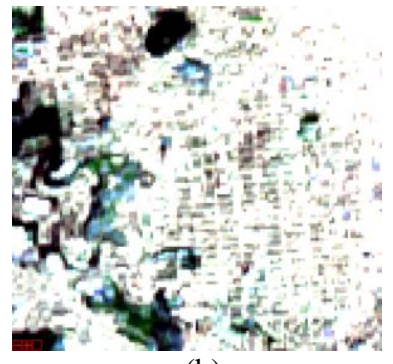

(b)

Fig. 4. Google earth (a), and Hyperspectral true color (b) images of study area

The study area includes mostly urban areas and forest areas. Google earth and hyperspectral true color images from the study area are shown in Fig.4.

\subsection{Built-Up Areas Detection}

The first to detect the built-up areas from POLSAR data, according to the Fig.1., we used the double bounce component of Freeman decomposition. The result is shown in Fig. 5. After further using the correlation coefficient in different polarization states by 5 degrees steps of ellipticity $(\tau)$ and orientation $(\varphi)$ angles, the refined built-up areas can be obtained as some of them shown in Fig. 6. Built-up areas detected in hyperspectral image by hCEM method using 5 adjusting parameter $(\lambda)$ and two of them are given in Fig.7.

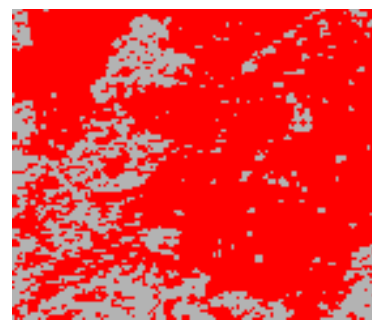

Fig. 5. Built-up areas estimated by using double bounce scattering component

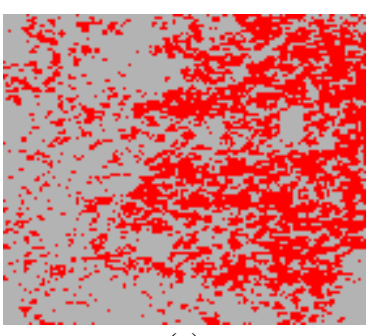

(a)

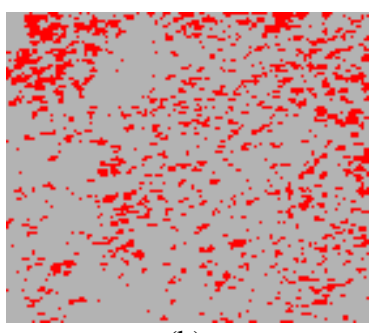

(b)

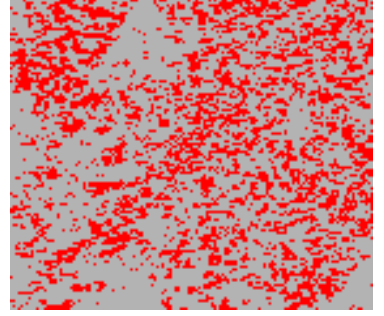

(c)

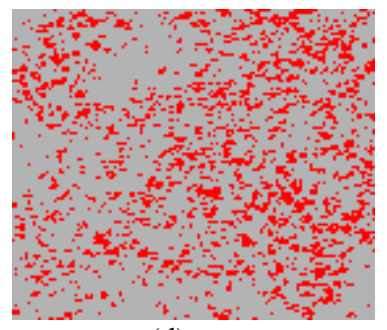

(d)
Fig. 6. Built-up areas estimated by using double bounce scattering component and the Correlation coefficient of (a) $\mathrm{HH}$, HV (b) $\tau=0, \varphi=100$ (c) $\tau=0, \varphi=135$ and (d) $\tau=-15, \varphi=55$ angles

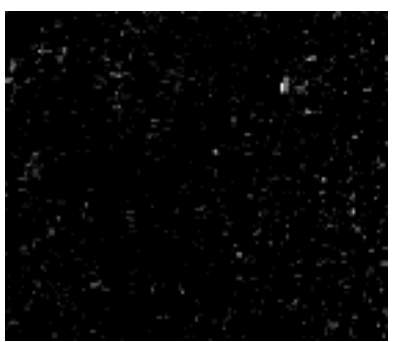

(a)

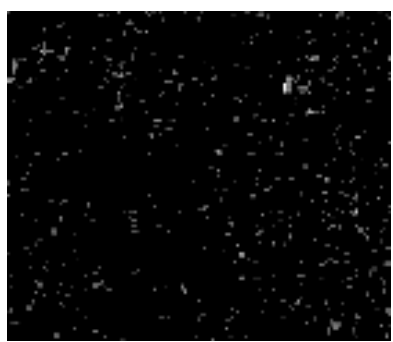

(b)
Fig. 7. Built-up areas detected by hCEM in two adjusting parameters, (a) 100 and (b) 10.

\subsection{Decision Fusion}

Different information sources can have different degrees of reliability, i.e., one data set might be more reliable than others in a specific analysis since the characteristics of sensors or data sets are not necessarily all the same. If each data set is taken as a separate information source, classification can be considered as an example of multisource data classification which has conceptually two different approaches.

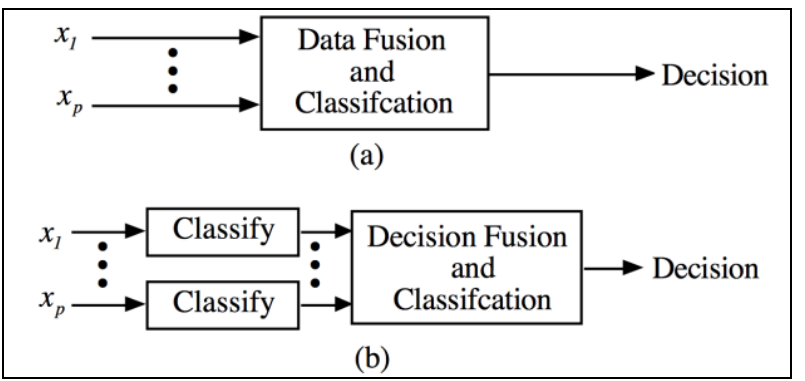

Fig. 8. Fusion procedures

One category is the data fusion approach shown in Fig.8(a) in which the feature vectors of the data sources (or sensor) are given to a central decision procedure which makes the final decision. The second category shown in Fig.8(b) is the decision fusion approach in which a final class decision is made by summarizing only the class decisions of each data set.

In this paper we used the second method by Majority voting procedure. As shown in Fig.9, the final target maps generated by majority voting on hyperspectral and POLSAR target detection maps, separately. If the pixel of two this maps is labeled as the target, then this pixel is determined as the target at the final built-up map as shown in Fig. 10. 


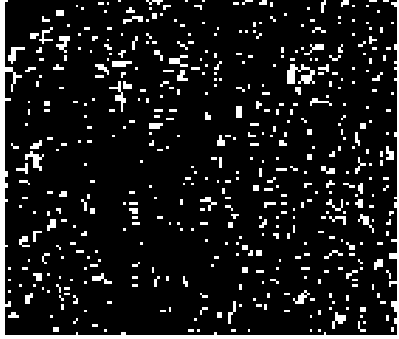

(a)

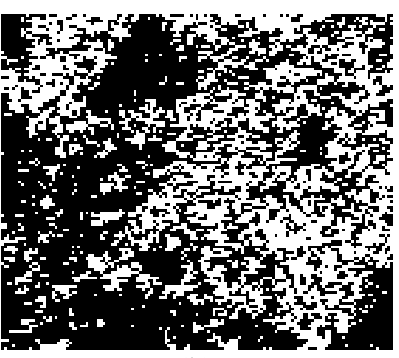

(b)
Fig. 9. Majority Voted Images of (a) Hyperspectral and (b) POLSAR

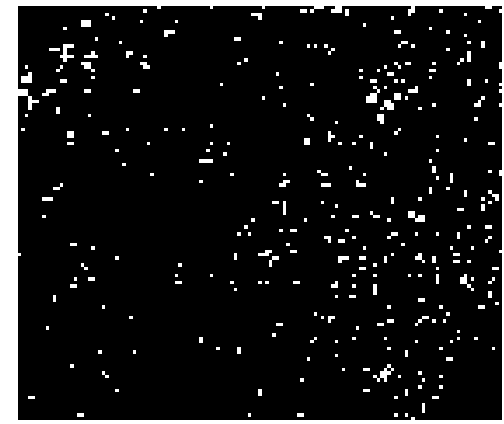

Fig. 10. Final Target map

Finally we calculated Correctness parameter $(\mathrm{Tp} / \mathrm{Tp}+\mathrm{Fp})$ for each output as shown in Table 1.

\begin{tabular}{|l|c|}
\hline Outputs & Correctness \\
\hline $\begin{array}{l}\text { Double bounce } \\
\text { scattering component }\end{array}$ & 0.57 \\
\hline $\begin{array}{l}\text { Double bounce } \\
\text { scattering component } \\
\text { and the Correlation } \\
\text { coefficient of HH, HV }\end{array}$ & 0.75 \\
\hline $\begin{array}{l}\text { Hyperspectral } \\
\text { component }\end{array}$ & 1.00 \\
\hline Fused Component & 1.00 \\
\hline
\end{tabular}

Table 1. Correctness parameters

\section{CONCLUSION}

When the resolution is low, for example, in the case of space borne SAR or vegetation such as tree or grass and building in the same resolution cell should be considered in realistic scattering scenario. In the first attempt we reserved the odd and even-bounce scattering components related to building and the remaining scattering components is removed. The built-up areas are estimated by using Freeman PTD and the correlation coefficients in several polarization states. On the other hand, and in second step we have used a hyperspectral target detection algorithm, the hCEM algorithm, which suppresses undesired background spectra and holds the target spectra through a layerby-layer filtering procedure and in each layer, we have constructed a better detector than previous layers. Experimental results on two real POLSAR and Hyperspectral images suggest that our procedures in POLSAR and Hyperspectral have reliable results.

\section{REFERENCES}

Alpers, W. (1983). "Imaging ocean surface waves by synthetic aperture radar: a review." Satellite microwave remote sensing: 107-119.

Cai, H., B. Zou, and M. Lin, "Parameter inversion models based on PolInSAR images," in Proc. APSAR 2007, Huangshan, China, 2007, pp. 751-754.

Chen, Y., N. M. Nasrabadi, and T. D. Tran, "Sparse representation for target detection in hyperspectral imagery," IEEE J. Sel. Topics Signal Process., vol. 5, no. 3, pp. 629-640, Jun. 2011.

Chen, Y., N. M. Nasrabadi, and T. D. Tran, "Simultaneous joint sparsity model for target detection in hyperspectral imagery," IEEE Geosci. Remote Sens. Lett., vol. 8, no. 4, pp. 676-680, Jul. 2011

Cloude, S. R. and K. P. Papathanassiou, "Polarimetric SAR interferometry,"IEEE Trans.Geosci. Remote Sens., vol. 36, no. 5, pp. 1551-1565, 1998.

Crisp, D. J. (2004). The state-of-the-art in ship detection in synthetic aperture radar imagery, DTIC Document.

Farrand, W. H. and J. C. Harsanyi (1997). "Mapping the distribution of mine tailings in the Coeur d'Alene River Valley, Idaho, through the use of a constrained energy minimization technique." Remote Sensing of Environment 59(1): 64-76.

Guillaso, S., L. Ferro-Famil, A. Reigber, and E. Pottier, "Building characterisation using polarimetric interferometric SAR data," in Proc. EUSAR, Ulm, Germany, May 25-27, 2004, pp. 407-410.

Guillaso, S., L. Ferro-Famil, A. Reigber, and E. Pottier, "Polarimetric interferometric SAR data analysis based on ESPRIT/MUSIC methods," presented at the POLINSAR'03, Frascati, Italy, Jan. 2003.

Guillaso, S., L. FerFamil, A. Reigber, and E. Pottier, "Building characterization using L-band polarimetric interferometric SAR data," IEEE Geosci. Remote Sens. Lett., vol. 2, no. 3, pp. 347 351, Jul. 2005.

Hajnsek, I. and S. R. Cloude, "Pol-InSAR for agriculture vegetation parameter estimation," in Proc. IGARSS'04, Sep. 20-24, 2004, vol. 2,pp. 1224-1227.

Harsanyi, J. C. and C.-I. Chang, "Hyperspectral image classification and dimensionality reduction: An orthogonal subspace projection approach," IEEE Trans. Geosci. Remote Sens., vol. 32, no. 4, pp. 779-785, Jul. 1994.

Kay, S. M. (1993). "Fundamentals of statistical signal processing: estimation theory." 
Kraut, S., L. L. Scharf and L. T. McWhorter (2001). "Adaptive subspace detectors." Signal Processing, IEEE Transactions on 49(1): 1-16.

Kruse, F. A. et al., "The spectral image processing system (SIPS) - Interactive visualization and analysis of imaging spectrometer data," Remote Sens. Environ., vol. 44, no. 2/3, pp. 145-163, May/Jun. 1993.

Manolakis, D. and G. Shaw (2002). "Detection algorithms for hyperspectral imaging applications." Signal Processing Magazine, IEEE 19(1): 29-43.

Manolakis, D., R. Lockwood, T. Cooley, and J. Jacobson, "Is there a best hyperspectral detection algorithm?" in Proc SPIE, Orlando, USA, 2009, pp. 1-16.

Manolakis, D., D. Marden, and G. A. Shaw, "Hyperspectral image processing for automatic target detection applications," Lincoln Lab. J., vol. 14, no. 1, pp. 79-116, 2003.

Manolakis, C. Siracusa, and G. Shaw, "Hyperspectral subpixel target detection using the linear mixing model," IEEE Trans. Geosci. Remote Sens., vol. 39, no. 7, pp. 1392-1409, Jul. 2001.

Moriyama, T. and S. Uratsuka et al., "Polarimetric SAR image analysis using model fit for urban structures," IEICE Trans. Commun., vol. E88-B, no. 3, pp. 1234-1242, 2005.

Nasrabadi, N. M. (2014). "Hyperspectral target detection: An overview of current and future challenges." Signal Processing Magazine, IEEE 31(1): 34-44.

Nunziata, F., M. Migliaccio and C. E. Brown (2012). "Reflection symmetry for polarimetric observation of man-made metallic targets at sea." Oceanic Engineering, IEEE Journal of 37(3): 384-394.

Papathanassiou, K. P. and S. R. Cloude, "Single baseline polarimetric SAR interferometry," IEEE Trans. Geosci. Remote Sens., vol. 39, no. 11, pp. 2352-2363, 2001.

Ren, H. and C.-I. Chang, "Target-constrained interference minimized approach to subpixel target detection for hyperspectral images," Opt. Eng., vol. 39, no. 12, pp. 31383145, Jul. 2000.

Rey, M. (2002). Results from the Crusade ship detection trial: Polarimetric SAR. Geoscience and Remote Sensing Symposium, 2002. IGARSS'02. 2002 IEEE International, IEEE.

Robey, F. C., D. R. Fuhrmann, E. J. Kelly, and R. Nitzberg, “A CFAR adaptive matched filter detector," IEEE Trans. Aerosp. Electron. Syst., vol. 28, no. 1, pp. 208-216, Jan. 1992.

Treuhaft, R. N., S. N.Madsen,M.Moghaddam, and J. J. van Zyl, "Vegetation characteristics and underlying topography from interferometric data," Radio Sci., vol. 31, pp. 1449-1495, 1996.

Treuhaft, R. N. and P. R. Siqueira, "The vertical structure of vegetated land surfaces from interferometric and polarimetric radar," Radio Sci., vol. 35, no. 1, pp. 141-177, 2000.

Treuhaft, R. N. and S. R. Cloude, "The structure of oriented vegetation from polarimetric interferometry," IEEE Trans. Geosci. Remote Sens., vol. 37, no. 5, pp. 2620-2624, 1999.
Tu, T.-M., C.-H. Chen, and C.-I. Chang, "A noise subspace projection approach to target signature detection and extraction in an unknown background for hyperspectral images," IEEE Trans. Geosci. Remote Sens., vol. 36, no. 1, pp. 171-181, Jan. 1998.

Wei, Y., Y. Wen, H. Sun, and M. S. Liao, "Unsupervised classification of PolInSAR data based on shannon entropy characterization with iterative optimization," IEEE J. Sel. Topics Appl. Earth Observ. Remote Sens. (JSTARS), vol. 4, no. 4, pp. 949-959, 2011.

Yamaguchi, Y. and T. Moriyama, "Four-component scattering model for polarimetric SAR image decomposition," IEEE Trans. Geosci. Remote Sens., vol. 43, no. 8, pp. 1699-1706, 2005.

Yang, S. and Z. Shi, "SparseCEM and SparseACE for hyperspectral image target detection," IEEE Geosci. Remote Sens. Lett., vol. 11, no. 12, pp. 2135-2139, Dec. 2014.

Zhang, L., B. Zou, H. Cai, and Y. Zhang, "Multiple-component scattering model for polarimetric SAR image decomposition," IEEE Geosci. Remote Sensing Lett., vol. 5, no. 4, pp. 603-607, Oct. 2008.

Zhang, L., L. Zhang, D. Tao, X. Huang, and B. Du, "Hyperspectral remote sensing image subpixel target detection based on supervised metric learning," IEEE Trans. Geosci. Remote Sens., vol. 52, no. 8, pp. 49554965, Aug. 2014.

Zou, Z.; Shi, Z., (2015). "Hierarchical Suppression Method for Hyperspectral Target Detection," in Geoscience and Remote Sensing, IEEE Transactions on, vol.PP, no.99, pp.1-13. 